\title{
Eigenvalues of the resistance-distance matrix of complete multipartite graphs
}

\author{
Kinkar Chandra Das ${ }^{1}$ and Yujun Yang ${ }^{2 *}$ (D)
}

\author{
"Correspondence: \\ yangyj@yahoo.com \\ ${ }^{2}$ School of Mathematics and \\ Information Science, Yantai \\ University, Yantai, 264005, P.R. China \\ Full list of author information is \\ available at the end of the article
}

\begin{abstract}
Let $G=(V, E)$ be a simple graph. The resistance distance between $i, j \in V$, denoted by $r_{i j}$, is defined as the net effective resistance between nodes $i$ and $j$ in the corresponding electrical network constructed from $G$ by replacing each edge of $G$ with a resistor of $1 \mathrm{Ohm}$. The resistance-distance matrix of $G$, denoted by $R(G)$, is a $|V| \times|V|$ matrix whose diagonal entries are 0 and for $i \neq j$, whose $i j$-entry is $r_{i j}$. In this paper, we determine the eigenvalues of the resistance-distance matrix of complete multipartite graphs. Also, we give some lower and upper bounds on the largest eigenvalue of the resistance-distance matrix of complete multipartite graphs. Moreover, we obtain a lower bound on the second largest eigenvalue of the resistance-distance matrix of complete multipartite graphs.
\end{abstract}

Keywords: resistance distance; resistance-distance matrix; largest resistance-distance eigenvalue; second largest resistance-distance eigenvalue

\section{Introduction}

Throughout the paper we consider only simple graphs, that is, graphs without loops and multi-edges. Let $G=(V, E)$ be a connected graph with a vertex set $V=\{1,2, \ldots, n\}$ and an edge set $E=E(G)$. The resistance distance [1] between any two vertices $i$ and $j$, denoted by $r_{i j}$, is defined as the net effective resistance between nodes $i$ and $j$ in the corresponding electrical network constructed from $G$ by replacing each edge with a resistor of $1 \mathrm{Ohm}$. The resistance-distance matrix of $G$, denoted by $R(G)$, is a $|V| \times|V|$ matrix whose diagonal entries are 0 and for $i \neq j$, whose $i j$-entry is $r_{i j}$. Let $\rho_{1} \geq \rho_{2} \geq \cdots \geq \rho_{n}$ denote the eigenvalues of $R(G)$. They are usually called the resistance-distance eigenvalues of $G$. In recent years, much study has been done on resistance distances. For more information, the readers are referred to most recent papers [2-12] and the references therein. In this paper, we study the resistance-distance matrix of complete multipartite graphs. The paper is organized as follows. In Section 2, we compute resistance distances in complete multipartite graphs. In Section 3, we determine the eigenvalues of the resistance distance matrix of complete multipartite graphs. In Section 4, we give some lower and upper bounds on the largest eigenvalue of the resistance-distance matrix of complete multipartite graphs. In Section 5, we obtain a lower bound on the second largest eigenvalue of the resistance-distance matrix of complete multipartite graphs.

(c) The Author(s) 2017. This article is distributed under the terms of the Creative Commons Attribution 4.0 International License (http://creativecommons.org/licenses/by/4.0/), which permits unrestricted use, distribution, and reproduction in any medium, provided you give appropriate credit to the original author(s) and the source, provide a link to the Creative Commons license, and indicate if changes were made. 


\section{Resistance distances in complete multipartite graphs}

In this section, we compute resistance distances between any pair of vertices in the complete multipartite graph $K_{n_{1}, n_{2}, \ldots, n_{k}}$ via electrical network approach. Recall that $G$ is a complete $k$-partite graph if the vertex set $V$ can be partitioned into $k$ parts $V_{1}, V_{2}, \ldots, V_{k}$ such that $u v \in E(G)$ if and only if $u$ and $v$ are in different parts. If $\left|V_{i}\right|=n_{i}(i=1,2, \ldots, k)$, then $G$ is denoted by $K_{n_{1}, n_{2}, \ldots, n_{k}}$. The following two lemmas play essential roles further.

Lemma 2.1 ([13]) Let $i, j$ be vertices of $G$ satisfying that they have the same neighborset $N$ in $V /\{i, j\}$. Then

$$
r_{i j}= \begin{cases}\frac{2}{|N|+2} & \text { if } i j \in E(G), \\ \frac{2}{|N|} & \text { otherwise. }\end{cases}
$$

Lemma 2.2 ([13] (The reduction principle)) If $S \subset V$ satisfies that all vertices in $S$ have the same neighborset $N$ in $G-S$. Let $H$ be the graph obtained from $G[S \cup N]$ by deleting all the edges between vertices in $N$. Then the resistance distance between any two vertices of $S$ in $G$ is the same as the resistance distance between them in $H$.

Now we are ready to give the main result of this section.

Theorem 2.3 Resistance distances in $K_{n_{1}, n_{2}, \ldots, n_{k}}$ can be computed as follows:

$$
r_{u v}= \begin{cases}\frac{2}{n-n_{i}} & \text { if } u, v \in V_{i}, \\ \frac{(n-1)\left(2 n-n_{i}-n_{j}\right)}{n\left[n^{2}-\left(n_{i}+n_{j}\right) n+n_{i} n_{j}\right]} & \text { if } u \in V_{i}, v \in V_{j}, \text { and } i \neq j .\end{cases}
$$

Proof For $u, v \in V_{i}$, it is easily seen that $u$ and $v$ have the same neighborset $N$ with $|N|=$ $n-n_{i}$. Hence, by Lemma 2.1, we have

$$
r_{u v}=\frac{2}{n-n_{i}}
$$

as required.

Now suppose that $u \in V_{i}$ and $v \in V_{j}$. Let $S=V_{i} \cup V_{j}$ and $N=V-S$. Let $G^{*}$ be the graph obtained from $K_{n_{1}, n_{2}, \ldots, n_{k}}$ by deleting all the edges between vertices in $N$. Then, by the reduction principle, the resistance distance between $u$ and $v$ in $K_{n_{1}, n_{2}, \ldots, n_{k}}$ is equal to the resistance distance between $u$ and $v$ in $G^{*}$. In what follows, we compute the resistance distance between $u$ and $v$ in $G^{*}$. If we apply a unit potential across $u$ and $v$, then by symmetry, all the vertices in $V_{i} \backslash\{u\}$ have the same potential, all the vertices in $V_{j} \backslash\{v\}$ have the same potential, and all the vertices in $N$ have the same potential. In an electrical point of view, vertices that have the same potential can be regarded as identical so that they can be shortened together. Consequently, we shorten all the vertices in $V_{i} \backslash\{u\}$ together to get a new vertex $x$, shorten all the vertices in $V_{j} \backslash\{v\}$ together to get a new vertex $y$, and shorten all the vertices in $N$ together to get a new vertex $w$. Then $G^{*}$ can be simplified to the network $\mathcal{N}$ as shown in Figure 1, where the weight $c_{a b}$ on each edge $a b$ denotes the edge conductance (i.e., reciprocal of edge resistance). 
Figure 1 The simplified graph $G^{*}$.

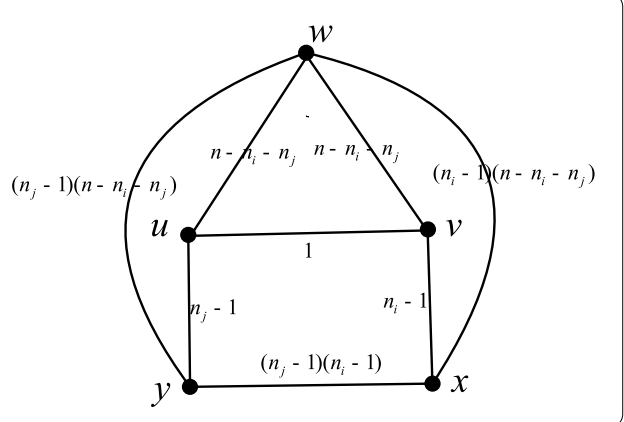

Now apply a unit potential across $u$ and $v$ in $\mathcal{N}$ and suppose the absolute potentials of $u, v, x, y, w$ are $V_{u}=1, V_{v}=0, V_{x}, V_{y}, V_{w}$, respectively. Then, by Kirchhoff's laws, we have

$$
\begin{aligned}
& \left(V_{x}-V_{y}\right) c_{x y}+\left(V_{x}-V_{v}\right) c_{x v}+\left(V_{x}-V_{w}\right) c_{x w}=0, \\
& \left(V_{y}-V_{x}\right) c_{x y}+\left(V_{y}-V_{u}\right) c_{y u}+\left(V_{y}-V_{w}\right) c_{y w}=0, \\
& \left(V_{w}-V_{u}\right) c_{w u}+\left(V_{w}-V_{x}\right) c_{x w}+\left(V_{w}-V_{y}\right) c_{y w}+\left(V_{w}-V_{v}\right) c_{w v}=0 .
\end{aligned}
$$

Then simple calculation shows that

$$
\begin{aligned}
V_{x} & =\frac{\left(n_{i}-1\right)\left(n_{j}-1\right) V_{y}+\left(n_{i}-1\right)\left(n-n_{i}-n_{j}\right) V_{w}}{n_{i}-1+\left(n_{i}-1\right)\left(n_{j}-1\right)+\left(n_{i}-1\right)\left(n-n_{i}-n_{j}\right)}, \\
V_{y} & =\frac{n_{j}-1+\left(n_{i}-1\right)\left(n_{j}-1\right) V_{x}+\left(n_{j}-1\right)\left(n-n_{i}-n_{j}\right) V_{w}}{n_{j}-1+\left(n_{i}-1\right)\left(n_{j}-1\right)+\left(n_{j}-1\right)\left(n-n_{i}-n_{j}\right)}, \\
V_{w} & =\frac{n-n_{i}-n_{j}+\left(n_{i}-1\right)\left(n-n_{i}-n_{j}\right) V_{x}+\left(n_{j}-1\right)\left(n-n_{i}-n_{j}\right) V_{y}}{2\left(n-n_{i}-n_{j}\right)+\left(n_{i}-1\right)\left(n-n_{i}-n_{j}\right)+\left(n_{j}-1\right)\left(n-n_{i}-n_{j}\right)} .
\end{aligned}
$$

Solving the above linear system, we get

$$
\begin{aligned}
V_{x} & =\frac{n\left(n_{j}-1\right)+(n-1)\left(n-n_{i}-n_{j}\right)}{\left(2 n-n_{i}-n_{j}\right)(n-1)}, \\
V_{y} & =\frac{n\left(n-n_{i}\right)}{\left(2 n-n_{i}-n_{j}\right)(n-1)}, \\
V_{w} & =\frac{n-n_{i}}{2 n-n_{i}-n_{j}} .
\end{aligned}
$$

Denote the total current flows from $u$ to $v$ by $I$. Then, by Ohm's law, we have

$$
\begin{aligned}
r_{u v} & =\frac{V_{u}-V_{v}}{I}=\frac{1-0}{I} \\
& =\frac{1}{\left(V_{u}-V_{w}\right)\left(n-n_{i}-n_{j}\right)+\left(V_{u}-V_{y}\right)\left(n_{j}-1\right)+\left(V_{u}-V_{v}\right) \times 1} \\
& =\frac{1}{\left(1-\frac{n-n_{i}}{2 n-n_{i}-n_{j}}\right)\left(n-n_{i}-n_{j}\right)+\left[1-\frac{n\left(n-n_{i}\right)}{\left(2 n-n_{i}-n_{j}\right)(n-1)}\right]\left(n_{j}-1\right)+1} \\
& =\frac{(n-1)\left(2 n-n_{i}-n_{j}\right)}{n\left[n^{2}-\left(n_{i}+n_{j}\right) n+n_{i} n_{j}\right]},
\end{aligned}
$$

as required. 
Remark It should be mentioned that resistance distances in complete multipartite graphs have also been determined in [10] via an alternative method.

By Theorem 2.3, for simplicity, in the following, we use $r_{i}$ to denote the resistance distance between any two vertices in $V_{i}$, and use $r_{i j}$ to denote the resistance distance between any $u \in V_{i}$ and $v \in V_{j}$. Thus the resistance-distance matrix $R\left(K_{n_{1}, n_{2}, \ldots, n_{k}}\right)$ of $K_{n_{1}, n_{2}, \ldots, n_{k}}$ is

$$
\left(\begin{array}{ccclc}
r_{1} J_{n_{1}, n_{1}}-r_{1} I_{n_{1}} & r_{12} J_{n_{1}, n_{2}} & r_{13} J_{n_{1}, n_{3}} & \ldots & r_{1 k} J_{n_{1}, n_{k}} \\
r_{12} J_{n_{2}, n_{1}} & r_{2} J_{n_{2}, n_{2}}-r_{2} I_{n_{2}} & r_{23} J_{n_{2}, n_{3}} & \ldots & r_{2 k} J_{n_{2}, n_{k}} \\
\vdots & \vdots & \vdots & \ldots & \vdots \\
r_{1 k} J_{n_{k}, n_{1}} & r_{2 k} J_{n_{k}, n_{2}} & r_{3 k} J_{n_{k}, n_{3}} & \ldots & r_{k} J_{n_{k}, n_{k}}-r_{k} I_{n_{k}}
\end{array}\right),
$$

where $J_{s t}$ denotes the $s \times t$ matrix of all ones, $I_{l}$ denotes the identity matrix of order $l$. In what follows, we always write $R\left(K_{n_{1}, n_{2}, \ldots, n_{k}}\right)$ to $R$ for short.

\section{The eigenvalues of the resistance-distance matrix of complete multipartite graphs}

In this section we obtain the eigenvalues of the resistance-distance matrix of complete $k$-partite graphs $K_{n_{1}, n_{2}, \ldots, n_{k}}$.

Theorem 3.1 Let $G$ be a complete $k$-partite graph $K_{n_{1}, n_{2}, \ldots, n_{k}}$ on $n$ vertices. Then the characteristic polynomial of $R$ is

$$
R_{G}(x)=\operatorname{det}\left(x I_{n}-R\right)=\prod_{i=1}^{k}\left(x+r_{i}\right)^{n_{i}-1}\left|x I_{k}-D_{2}\right|,
$$

where

$$
D_{2}=\left(\begin{array}{ccccc}
\left(n_{1}-1\right) r_{1} & n_{2} r_{12} & n_{3} r_{13} & \cdots & n_{k} r_{1 k} \\
n_{1} r_{12} & \left(n_{2}-1\right) r_{2} & n_{3} r_{23} & \cdots & n_{k} r_{2 k} \\
\vdots & \vdots & \vdots & \ddots & \vdots \\
n_{1} r_{1 k} & n_{2} r_{2 k} & n_{3} r_{3 k} & \cdots & \left(n_{k}-1\right) r_{k}
\end{array}\right)
$$

Proof As given above, the resistance-distance matrix of $K_{n_{1}, n_{2}, \ldots, n_{k}}$ is

$$
R=\left(\begin{array}{ccccc}
r_{1} J_{n_{1}, n_{1}}-r_{1} I_{n_{1}} & r_{12} J_{n_{1}, n_{2}} & r_{13} J_{n_{1}, n_{3}} & \ldots & r_{1 k} J_{n_{1}, n_{k}} \\
r_{12} J_{n_{2}, n_{1}} & r_{2} J_{n_{2}, n_{2}}-r_{2} I_{n_{2}} & r_{23} J_{n_{2}, n_{3}} & \ldots & r_{2 k} J_{n_{2}, n_{k}} \\
\vdots & \vdots & \vdots & \ldots & \vdots \\
r_{1 k} J_{n_{k}, n_{1}} & r_{2 k} J_{n_{k}, n_{2}} & r_{3 k} J_{n_{k}, n_{3}} & \ldots & r_{k} J_{n_{k}, n_{k}}-r_{k} I_{n_{k}}
\end{array}\right)
$$

Hence, by linear algebra knowledge,

$$
\begin{aligned}
R_{G}(x) & =\operatorname{det}\left(x I_{n}-R\right) \\
& =\left|\begin{array}{ccccc}
\left(x+r_{1}\right) I_{n_{1}}-r_{1} J_{n_{1}, n_{1}} & -r_{12} J_{n_{1}, n_{2}} & -r_{13} J_{n_{1}, n_{3}} & \ldots & -r_{1 k} J_{n_{1}, n_{k}} \\
-r_{12} J_{n_{2}, n_{1}} & \left(x+r_{2}\right) I_{n_{2}}-r_{2} J_{n_{2}, n_{2}} & -r_{23} J_{n_{2}, n_{3}} & \ldots & -r_{2 k} J_{n_{2}, n_{k}} \\
\vdots & \vdots & \vdots & \ldots & \vdots \\
-r_{1 k} J_{n_{k}, n_{1}} & -r_{2 k} J_{n_{k}, n_{2}} & -r_{3 k} J_{n_{k}, n_{3}} & \ldots & \left(x+r_{k}\right) I_{n_{k}}-r_{k} J_{n_{k}, n_{k}}
\end{array}\right|
\end{aligned}
$$




$$
=\prod_{i=1}^{k}\left(x+r_{i}\right)^{n_{i}-1}\left|\begin{array}{ccccc}
x-\left(n_{1}-1\right) r_{1} & -n_{2} r_{12} & -n_{3} r_{13} & \cdots & -n_{k} r_{1 k} \\
-n_{1} r_{12} & x-\left(n_{2}-1\right) r_{2} & -n_{3} r_{23} & \cdots & -n_{k} r_{2 k} \\
\vdots & \vdots & \vdots & \ddots & \vdots \\
-n_{1} r_{1 k} & -n_{2} r_{2 k} & -n_{3} r_{3 k} & \cdots & x-\left(n_{k}-1\right) r_{k}
\end{array}\right| .
$$

This completes the proof.

Corollary 3.2 Let $G=K_{n_{1}, n_{2}, \ldots, n_{k}}$. Then the eigenvalues for the resistance-distance matrix of $G$ are $-r_{i}$ of multiplicity $n_{i}-1(i=1,2, \ldots, k)$ and the remaining eigenvalues satisfy the following:

$$
\left|x I_{k}-D_{2}\right|=0
$$

where $D_{2}$ is given by (6).

Corollary 3.3 The largest eigenvalue of the resistance-distance matrix of complete $k$ partite graph $K_{n_{1}, n_{2}, \ldots, n_{k}}$ is given by

$$
\left|x I_{k}-D_{2}\right|=0 \text {. }
$$

\section{Lower and upper bounds on the largest eigenvalue of the}

\section{resistance-distance matrix of complete multipartite graphs}

In this section we give some lower and upper bounds on $\rho_{1}(G)$ of complete $k$-partite graph $K_{n_{1}, n_{2}, \ldots, n_{k}}$. For this we need the following two results.

Lemma 4.1 ([14]) If $q_{1}, q_{2}, \ldots, q_{n}$ are positive numbers, then

$$
\min _{i} \frac{p_{i}}{q_{i}} \leq \frac{p_{1}+p_{2}+\cdots+p_{n}}{q_{1}+q_{2}+\cdots+q_{n}} \leq \max _{i} \frac{p_{i}}{q_{i}}
$$

for any real numbers $p_{1}, p_{2}, \ldots, p_{n}$. Equality holds on both sides if and only if all the ratios $\frac{p_{i}}{q_{i}}$ are equal.

Now we obtain the spectral radius for the resistance-distance matrix of $K_{n_{1}, n_{1}, \ldots, n_{1}}$.

Lemma 4.2 Let $G$ be a complete $k$-partite graph $K_{n_{1}, n_{1}, \ldots, n_{1}}$ of order $n$. Then

$$
\rho_{1}(G)=\frac{2}{n-n_{1}}\left[n_{1}-1+\frac{n_{1}(k-1)(n-1)}{n}\right] \text {. }
$$

Furthermore, all the remaining eigenvalues are $-\frac{2}{n-n_{1}}$ and $-\frac{2}{n}$, with multiplicities $n-k$ and $k-1$, respectively.

Proof For $G=K_{n_{1}, n_{1}, \ldots, n_{1}}$, by Theorem 2.3, we have $r_{i}=\frac{2}{n-n_{1}}(i=1,2, \ldots, k)$ and $r_{i j}=$ $\frac{n-1}{n}\left(\frac{1}{n-n_{1}}+\frac{1}{n-n_{1}}\right)$ for all $i$ and $j, i \neq j$. Since

$$
D_{2}=\left(\begin{array}{ccccc}
\left(n_{1}-1\right) r_{1} & n_{1} r_{12} & n_{1} r_{12} & \cdots & n_{1} r_{12} \\
n_{1} r_{12} & \left(n_{1}-1\right) r_{1} & n_{1} r_{12} & \cdots & n_{1} r_{12} \\
\vdots & \vdots & \vdots & \ddots & \vdots \\
n_{1} r_{12} & n_{1} r_{12} & n_{1} r_{12} & \cdots & \left(n_{1}-1\right) r_{1}
\end{array}\right)
$$


it is easily obtained that the eigenvalues of $D_{2}$ are

$$
\left(n_{1}-1\right) r_{1}+(k-1) r_{12}, \underbrace{\left(n_{1}-1\right) r_{1}-n_{1} r_{12}, \ldots,\left(n_{1}-1\right) r_{1}-n_{1} r_{12}}_{k-1} .
$$

Simple calculation shows that

$$
\left(n_{1}-1\right) r_{1}+(k-1) r_{12}=\frac{2}{n-n_{1}}\left[n_{1}-1+\frac{n_{1}(k-1)(n-1)}{n}\right] \text { and } \quad\left(n_{1}-1\right) r_{1}-n_{1} r_{12}=-\frac{2}{n} .
$$

Thus it follows that

$$
\rho_{1}(G)=\frac{2}{n-n_{1}}\left[n_{1}-1+\frac{n_{1}(k-1)(n-1)}{n}\right] .
$$

Together with the result in Theorem 2.3, we conclude that eigenvalues other than $\rho_{1}(G)$ are

$$
\underbrace{-\frac{2}{n}, \ldots,-\frac{2}{n}}_{k-1}, \underbrace{-\frac{2}{n-n_{1}}, \ldots,-\frac{2}{n-n_{1}}}_{n-k} .
$$

Theorem 4.3 Let $G$ be a complete $k$-partite graph $K_{n_{1}, n_{2}, \ldots, n_{k}}$ of order $n$ with $n_{1} \geq n_{2} \geq$ $\cdots \geq n_{k}$. Then

$$
\frac{2}{n-n_{k}}\left[n_{k}-1+\frac{n_{k}(k-1)(n-1)}{n}\right] \leq \rho_{1}(G) \leq \frac{2}{n-n_{1}}\left[n_{1}-1+\frac{n_{1}(k-1)(n-1)}{n}\right] .
$$

Moreover, the equality holds in both sides if and only if $n_{1}=n_{2}=\cdots=n_{k}$.

Proof Let $\mathbf{x}=\left(x_{1}, x_{2}, \ldots, x_{n}\right)^{T}$ be an eigenvector corresponding to the eigenvalue $\rho_{1}(G)$ of $D_{2}$. Then we have

$$
D_{2} \mathbf{x}=\rho_{1}(G) \mathbf{x}
$$

From the $i$ th equation of (8), we get

$$
\begin{aligned}
\rho_{1}(G) x_{i} & =\left(n_{i}-1\right) r_{i} x_{i}+\sum_{j=1, j \neq i}^{k} n_{j} r_{i j} x_{j} \\
& =\frac{2\left(n_{i}-1\right)}{n-n_{i}} x_{i}+\frac{n-1}{n} \sum_{j=1, j \neq i}^{k}\left[\frac{1}{n-n_{i}}+\frac{1}{n-n_{j}}\right] n_{j} x_{j} \\
& \geq \frac{2\left(n_{k}-1\right)}{n-n_{k}} x_{i}+\frac{2(n-1)}{n\left(n-n_{k}\right)} \sum_{j=1, j \neq i}^{k} n_{j} x_{j} \quad \text { as } n_{i}, n_{j} \geq n_{k} .
\end{aligned}
$$

Taking summation on both sides from $i=1$ to $k$, we get

$$
\rho_{1}(G) \sum_{i=1}^{k} x_{i} \geq \frac{2\left(n_{k}-1\right)}{n-n_{k}} \sum_{i=1}^{k} x_{i}+\frac{2(n-1)(k-1)}{n\left(n-n_{k}\right)} \sum_{i=1}^{k} n_{i} x_{i} .
$$


By Lemma 4.1, we get

$$
\frac{\sum_{i=1}^{k} n_{i} x_{i}}{\sum_{i=1}^{k} x_{i}} \geq n_{k}
$$

From the above two results, we get

$$
\rho_{1}(G) \geq \frac{2\left(n_{k}-1\right)}{n-n_{k}}+\frac{2(n-1)(k-1)}{n\left(n-n_{k}\right)} n_{k}=\frac{2}{n-n_{k}}\left[n_{k}-1+\frac{n_{k}(k-1)(n-1)}{n}\right] .
$$

Since $n_{i}, n_{j} \leq n_{1}$ and

$$
\frac{\sum_{i=1}^{k} n_{i} x_{i}}{\sum_{i=1}^{k} x_{i}} \leq n_{1}
$$

similarly, from the above, we get

$$
\rho_{1}(G) \leq \frac{2}{n-n_{1}}\left[n_{1}-1+\frac{n_{1}(k-1)(n-1)}{n}\right]
$$

First part of the proof is done.

Now suppose that the left-hand side equality holds in (7). Then all the inequalities above must be equalities. From the equality in (9), we get $n_{1}=n_{2}=\cdots=n_{k}$. From the equality in (10), we get $n_{1}=n_{2}=\cdots=n_{k}$. Similarly, if the right-hand side equality holds in (7), then we have $n_{1}=n_{2}=\cdots=n_{k}$.

Conversely, let $G \cong K_{n_{1}, n_{1}, \ldots, n_{1}}$. By Lemma 4.2 , equalities on both sides hold in (7).

Now we give another upper bound on $\rho_{1}(G)$ of complete $k$-partite graph $K_{n_{1}, n_{2}, \ldots, n_{k}}$.

Theorem 4.4 Let $G$ be a complete k-partite graph $K_{n_{1}, n_{2}, \ldots, n_{k}}$ of order $n$ with $n_{1} \geq n_{2} \geq$ $\cdots \geq n_{k}$. Then

$$
\begin{aligned}
\rho_{1}(G) \leq & \frac{2\left(n_{1}-1\right)}{n-n_{1}}+\frac{n_{1}(n-1)^{2}(k-1)}{n\left(n-n_{1}\right)\left(n_{k}-1\right)}-\frac{(n-1)\left(n-n_{1}\right)}{n\left(n_{1}-1\right)} \\
& +\frac{(n-1)\left(n-n_{k}\right)(k-1)}{n\left(n_{k}-1\right)}\left[1-\frac{(2 n-1)}{n-n_{1}}+\frac{n(n-1)}{\left(n-n_{1}\right)^{2}}\right]
\end{aligned}
$$

with equality holding if and only if $n_{1}=n_{2}=\cdots=n_{k}$.

Proof Let $\mathbf{x}=\left(x_{1}, x_{2}, \ldots, x_{n}\right)^{T}$ be an eigenvector corresponding to the eigenvalue $\rho_{1}(G)$ of $C^{-1} D_{2} C$, where $C=\operatorname{diag}\left(\left(n_{1}-1\right) r_{1},\left(n_{2}-1\right) r_{2}, \ldots,\left(n_{k}-1\right) r_{k}\right)$. Then we have

$$
C^{-1} D_{2} C \mathbf{X}=\rho_{1}(G) \mathbf{X}
$$

We can assume that $x_{i}=1$ and $x_{k} \leq 1$ for all $k$. From the $i$ th equation of (12), we get

$$
\rho_{1}(G) x_{i}=\left(n_{i}-1\right) r_{i} x_{i}+\sum_{j=1, j \neq i}^{k} \frac{\left(n_{j}-1\right) r_{j} n_{j} r_{i j}}{\left(n_{i}-1\right) r_{i}} x_{j}
$$


that is,

$$
\begin{aligned}
\rho_{1}(G) & \leq\left(n_{i}-1\right) r_{i}+\sum_{j=1, j \neq i}^{k} \frac{\left(n_{j}-1\right) r_{j} n_{j} r_{i j}}{\left(n_{i}-1\right) r_{i}} \\
& =\frac{2\left(n_{i}-1\right)}{n-n_{i}}+\sum_{j=1, j \neq i}^{k} \frac{(n-1)\left(n_{j}-1\right) n_{j}}{n\left(n_{i}-1\right)}\left[\frac{1}{n-n_{j}}+\frac{n-n_{i}}{\left(n-n_{j}\right)^{2}}\right] .
\end{aligned}
$$

Since

$$
\frac{n_{j}\left(n_{j}-1\right)}{n-n_{j}}=1-n-n_{j}+\frac{n(n-1)}{n-n_{j}}
$$

and

$$
\frac{n_{j}\left(n_{j}-1\right)}{\left(n-n_{j}\right)^{2}}=1-\frac{2 n-1}{n-n_{j}}+\frac{n(n-1)}{\left(n-n_{j}\right)^{2}}
$$

from (14), we get

$$
\begin{aligned}
\rho_{1}(G) \leq & \frac{2\left(n_{i}-1\right)}{n-n_{i}}+\frac{n-1}{n\left(n_{i}-1\right)} \sum_{j=1, j \neq i}^{k}\left[1-n-n_{j}+\frac{n(n-1)}{n-n_{j}}\right]+\frac{(n-1)\left(n-n_{i}\right)}{n\left(n_{i}-1\right)} \\
& \times \sum_{j=1, j \neq i}^{k}\left[1-\frac{2 n-1}{n-n_{j}}+\frac{n(n-1)}{\left(n-n_{j}\right)^{2}}\right] .
\end{aligned}
$$

Since

$$
f(x)=\frac{n(n-1)}{(n-x)^{2}}-\frac{2 n-1}{(n-x)}
$$

is an increasing function on $x$, from the above, we get

$$
\begin{aligned}
\rho_{1}(G) \leq & \frac{2\left(n_{i}-1\right)}{n-n_{i}}+\frac{n-1}{n\left(n_{i}-1\right)}\left[-(n-1)(k-1)-\left(n-n_{i}\right)+\frac{n(n-1)(k-1)}{n-n_{1}}\right] \\
& +\frac{(n-1)\left(n-n_{i}\right)}{n\left(n_{i}-1\right)}\left[1-\frac{2 n-1}{n-n_{1}}+\frac{n(n-1)}{\left(n-n_{1}\right)^{2}}\right](k-1) \\
= & \frac{2\left(n_{i}-1\right)}{n-n_{i}}+\frac{n_{1}(n-1)^{2}(k-1)}{n\left(n-n_{1}\right)\left(n_{i}-1\right)}-\frac{(n-1)\left(n-n_{i}\right)}{n\left(n_{i}-1\right)} \\
& +\frac{(n-1)\left(n-n_{i}\right)(k-1)}{n\left(n_{i}-1\right)}\left[1-\frac{(2 n-1)}{n-n_{1}}+\frac{n(n-1)}{\left(n-n_{1}\right)^{2}}\right] .
\end{aligned}
$$

Let us consider a function

$$
g(x)=1-\frac{(2 n-1)}{n-x}+\frac{n(n-1)}{(n-x)^{2}}, \quad 1 \leq x \leq n-1 .
$$

Then

$$
g^{\prime}(x)=\frac{1}{(n-x)^{3}}[(2 n-1) x-n]>0 .
$$


Therefore $g(x) \geq g(1)=0$. Since

$$
\frac{n-n_{1}}{n_{1}-1} \leq \frac{n-n_{i}}{n_{i}-1} \leq \frac{n-n_{k}}{n_{k}-1}
$$

and $n_{k} \leq n_{i} \leq n_{1}(1 \leq i \leq k)$, from (15), we get

$$
\begin{aligned}
\rho_{1}(G) \leq & \frac{2\left(n_{1}-1\right)}{n-n_{1}}+\frac{n_{1}(n-1)^{2}(k-1)}{n\left(n-n_{1}\right)\left(n_{k}-1\right)}-\frac{(n-1)\left(n-n_{1}\right)}{n\left(n_{1}-1\right)} \\
& +\frac{(n-1)\left(n-n_{k}\right)(k-1)}{n\left(n_{k}-1\right)}\left[1-\frac{(2 n-1)}{n-n_{1}}+\frac{n(n-1)}{\left(n-n_{1}\right)^{2}}\right],
\end{aligned}
$$

which gives the required result in (11). First part of the proof is done.

Now suppose that equality holds in (11). Then all the inequalities above must be equalities. From equality in (13), we get $x_{1}=x_{2}=\cdots=x_{k}$. From equality in (15), we get $n_{1}=n_{2}=$ $\cdots=n_{i-1}=n_{i+1}=\cdots=n_{k}$. From equality in (16), we get $n_{i}=n_{1}=n_{k}$. From these results we conclude that $n_{1}=n_{2}=\cdots=n_{k}$.

Conversely, let $n_{1}=n_{2}=\cdots=n_{k}$. Then $n=n_{1} k$. Now,

$$
\begin{aligned}
& \frac{2\left(n_{1}-1\right)}{n-}+\frac{n_{1}(n-1)^{2}(k-1)}{n\left(n-n_{1}\right)\left(n_{1}-1\right)}-\frac{(n-1)\left(n-n_{1}\right)}{n\left(n_{1}-1\right)}+\frac{(n-1)\left(n-n_{1}\right)(k-1)}{n\left(n_{1}-1\right)} \\
& \quad \times\left[1-\frac{(2 n-1)}{n-n_{1}}+\frac{n(n-1)}{\left(n-n_{1}\right)^{2}}\right] \\
& =\frac{2\left(n_{1}-1\right)}{n-n_{1}}+\frac{(n-1)^{2}}{n\left(n_{1}-1\right)}-\frac{(n-1)\left(n-n_{1}\right)}{n\left(n_{1}-1\right)}+\frac{(n-1)(k-1)^{2}}{k\left(n_{1}-1\right)} \times \frac{n_{1}\left(n_{1}-1\right)}{\left(n-n_{1}\right)^{2}} \\
& =\frac{2\left(n_{1}-1\right)}{n-n_{1}}+\frac{2(n-1)}{n}=\rho_{1}(G), \quad \text { by Lemma } 4.2 .
\end{aligned}
$$

\section{Lower bound on the second largest eigenvalue of the resistance-distance matrix of complete multipartite graphs}

In this section we find a lower bound on the second largest eigenvalue of the resistancedistance matrix of complete multipartite graphs. For this we need the following result.

Lemma 5.1 ([15]) Let $A$ be a $p \times p$ symmetric matrix, and let $A_{k}$ be its leading $k \times k$ submatrix; that is, $A_{k}$ is the matrix obtained from $A$ by deleting its last $p-k$ rows and columns. Then, for $i=1,2, \ldots, k$,

$$
\lambda_{p-i+1}(A) \leq \lambda_{k-i+1}\left(A_{k}\right) \leq \lambda_{k-i+1}(A)
$$

where $\lambda_{i}(A)$ is the ith largest eigenvalue of $A$.

Theorem 5.2 Let $G$ be a complete k-partite graph $K_{n_{1}, n_{2}, \ldots, n_{k}}$ of order $n$ with $n_{1} \geq n_{2} \geq$ $\cdots \geq n_{k}$. Then

$$
\begin{aligned}
\rho_{2}(G) \geq & \max _{1 \leq i<j \leq k}\left[\frac{(n+1)\left(n_{i}+n_{j}\right)-2\left(n+n_{i} n_{j}\right)}{\left(n-n_{i}\right)\left(n-n_{j}\right)}-\frac{(n-1)\left(n_{i}+n_{j}\right)}{\left(n-n_{i}\right)\left(n-n_{j}\right)}\right. \\
& \times \sqrt{\left.\left(1+\frac{n_{i} n_{j}}{n^{2}}\right)-\frac{4 n_{i} n_{j}}{n\left(n_{i}+n_{j}\right)}\right]} .
\end{aligned}
$$


Proof By Lemma 5.1, we have $\rho_{1}(G) \geq \max _{1 \leq i<j \leq k} \rho_{1}^{\prime}$ and $\rho_{2}(G) \geq \max _{1 \leq i<j \leq k} \rho_{2}^{\prime}$, where $\rho_{1}^{\prime}$ and $\rho_{2}^{\prime}$ are given by

$$
\left|\begin{array}{cc}
\left(n_{i}-1\right) r_{i}-\rho & n_{j} r_{i j} \\
n_{i} r_{i j} & \left(n_{j}-1\right) r_{j}-\rho
\end{array}\right|=0
$$

that is,

$$
\rho^{2}-\left[\left(n_{i}-1\right) r_{i}+\left(n_{j}-1\right) r_{j}\right] \rho+\left(n_{i}-1\right)\left(n_{j}-1\right) r_{i} r_{j}-n_{i} n_{j} r_{i j}^{2}=0
$$

So,

$$
\rho_{1}^{\prime}=\frac{\left(n_{i}-1\right) r_{i}+\left(n_{j}-1\right) r_{j}+\sqrt{\left[\left(n_{i}-1\right) r_{i}-\left(n_{j}-1\right) r_{j}\right]^{2}+4 n_{i} n_{j} r_{i j}^{2}}}{2}
$$

and

$$
\rho_{2}^{\prime}=\frac{\left(n_{i}-1\right) r_{i}+\left(n_{j}-1\right) r_{j}-\sqrt{\left[\left(n_{i}-1\right) r_{i}-\left(n_{j}-1\right) r_{j}\right]^{2}+4 n_{i} n_{j} r_{i j}^{2}}}{2} .
$$

Now,

$$
\begin{aligned}
\left(n_{i}-1\right) r_{i}-\left(n_{j}-1\right) r_{j} & =\frac{2\left(n_{i}-1\right)}{n-n_{i}}-\frac{2\left(n_{j}-1\right)}{n-n_{j}} \\
& =\frac{2(n-1)\left(n_{i}-n_{j}\right)}{\left(n-n_{i}\right)\left(n-n_{j}\right)} .
\end{aligned}
$$

Using the above result, we get

$$
\begin{aligned}
& {\left[\left(n_{i}-1\right) r_{i}-\left(n_{j}-1\right) r_{j}\right]^{2}+4 n_{i} n_{j} r_{i j}^{2}} \\
& \quad=\frac{4(n-1)^{2}\left(n_{i}-n_{j}\right)^{2}}{\left(n-n_{i}\right)^{2}\left(n-n_{j}\right)^{2}}+\frac{4 n_{i} n_{j}(n-1)^{2}}{n^{2}}\left(\frac{1}{n-n_{i}}+\frac{1}{n-n_{j}}\right)^{2} \\
& \quad=\frac{4(n-1)^{2}\left(n_{i}+n_{j}\right)^{2}}{\left(n-n_{i}\right)^{2}\left(n-n_{j}\right)^{2}}\left[\left(1+\frac{n_{i} n_{j}}{n^{2}}\right)-\frac{4 n_{i} n_{j}}{n\left(n_{i}+n_{j}\right)}\right] .
\end{aligned}
$$

Moreover,

$$
\begin{aligned}
\left(n_{i}-1\right) r_{i}+\left(n_{j}-1\right) r_{j} & =\frac{2\left(n_{i}-1\right)}{n-n_{i}}+\frac{2\left(n_{j}-1\right)}{n-n_{j}} \\
& =\frac{2\left[(n+1)\left(n_{i}+n_{j}\right)-2\left(n+n_{i} n_{j}\right)\right]}{\left(n-n_{i}\right)\left(n-n_{j}\right)} .
\end{aligned}
$$

Using (20) and (21) in (19), we get the required result in (18).

Corollary 5.3 Let $G$ be a complete $k$-partite graph $K_{n_{1}, n_{2}, \ldots, n_{k}}$ of order $n$ with $n_{1} \geq n_{2} \geq$ $\cdots \geq n_{k}$. Then

$$
\rho_{2}(G) \geq \frac{(n+1)\left(n_{1}+n_{2}\right)-2\left(n+n_{1} n_{2}\right)}{\left(n-n_{1}\right)\left(n-n_{2}\right)}-\frac{(n-1)\left(n_{1}+n_{2}\right)}{\left(n-n_{1}\right)\left(n-n_{2}\right)} \sqrt{\left(1+\frac{n_{1} n_{2}}{n^{2}}\right)-\frac{4 n_{1} n_{2}}{n\left(n_{1}+n_{2}\right)}} .
$$




\section{Conclusion}

In this paper, resistance distances in complete multipartite graphs are given via the standard electrical approach. Then eigenvalues of the resistance-distance matrix of complete multipartite graphs are studied, with emphasis being placed on bounds for the largest and second largest eigenvalues. However, up to now, the study on the eigenvalues of a resistance-distance matrix has still been in its infancy. Further study in this field is greatly anticipated.

\section{Acknowledgements}

We would like to thank the anonymous referees for their careful review and valuable comments. The second author was supported by the National Science Foundation of China through grant no. 11671347 and Yantai University Foundation through grant no. SX14GG3.

\section{Competing interests}

The authors declare that they have no competing interests.

\section{Authors' contributions}

All the authors contributed equally to this work. All authors read and approved the final manuscript.

\section{Author details}

'Department of Mathematics, Sungkyunkwan University, Suwon, 440-746, Republic of Korea. ${ }^{2}$ School of Mathematics and Information Science, Yantai University, Yantai, 264005, P.R. China.

\section{Publisher's Note}

Springer Nature remains neutral with regard to jurisdictional claims in published maps and institutional affiliations.

Received: 2 May 2017 Accepted: 17 November 2017 Published online: 28 November 2017

\section{References}

1. Klein, DJ, Randić, M: Resistance distance. J. Math. Chem. 12, 81-95 (1993)

2. Bu, C, Yan, B, Zhou, X, Zhou, J: Resistance distance in subdivision-vertex join and subdivision-edge join of graphs. Linear Algebra Appl. 458, 454-462 (2014)

3. Tan, Z, Essam, JW, Wu, F: Two-point resistance of a resistor network embedded on a globe. Phys. Rev. E 90,012130 (2014)

4. Yang, Y, Klein, DJ: Resistance distances in composite graphs. J. Phys. A, Math. Theor. 47, 375203 (2014)

5. Liu, J, Cao, J: The resistance distances of electrical networks based on Laplacian generalized inverse. Neurocomputing 167, 306-313 (2015)

6. Liu, X, Zhou, J, Bu, C: Resistance distance and Kirchhoff index of R-vertex join and R-edge join of two graphs. Discrete Appl. Math. 187, 130-139 (2015)

7. Sun, L, Wang, W, Zhou, J, Bu, C: Some results on resistance distances and resistance matrices. Linear Multilinear Algebra 63, 523-533 (2015)

8. Tan, Z: Recursion-transform method to a non-regular $m \times n$ cobweb with an arbitrary longitude. Sci. Rep. 5, 11266 (2015)

9. Tan, Z, Fang, J: Two-point resistance of a cobweb network with a 2 r boundary. Commun. Theor. Phys. 63, 36-44 (2015)

10. Gervacio, SV: Resistance distance in complete n-partite graphs. Discrete Appl. Math. 203, 53-61 (2016)

11. Huang, Q, Chen, H, Deng, Q: Resistance distances and the Kirchhoff index in double graphs. J. Appl. Math. Comput. 50, 1-14 (2016)

12. Zhou, J, Wang, Z, Bu, C: On the resistance matrix of a graph. Electron. J. Comb. 23(1), P1.41 (2016)

13. Yang, Y, Zhang, H: Some rules on resistance distance with applications. J. Phys. A, Math. Theor. 41, 445203 (2008)

14. Papendieck, B, Recht, P: On maximal entries in the principal eigenvector of graphs. Linear Algebra Appl. 310, 129-138 (2010)

15. Schott, JR: Matrix Analysis for Statistics. John Wiley and Sons, New York (1997) 